\title{
Modeling and Imaging Interactive Biomaterial Effects on Self-Organizing Tissues
}

Jay D. Potts ${ }^{1}$, Lorain Junor ${ }^{1}$, Austin Worden ${ }^{1}$ and Mark Uline ${ }^{2}$

1. Department of Cell Biology and Anatomy, University of South Carolina, School of Medicine, Columbia, SC.

2. Instrumentation Resource Facility, University of South Carolina School of Medicine, Columbia, SC.

3. Department of Chemical Engineering, University of South Carolina, Columbia, SC.

It has become evident that the use of three-dimensional (3D) culture systems provides a distinct advantage over 2D systems when examining how cells interact in their natural environment. Recent work has demonstrated the changes in cellular characteristics such as morphology, speed and directionality of cells when both planar and 3D matrices are compared [1]. The way that cell migrate is dependent on a number of variables such as cellular content of the substrate, the physical environment of the substrate and the chemical milieu of the surrounding matrix, to name just a few [2-4]. In our previous work, we investigated the advantages of using collagen hydrogels as $3 \mathrm{D}$ scaffolds for culturing cells ${ }^{5}$. Interactions between collagen hydrogels and epithelial cells were observed, and it was found that placing the cells on top of the gels as compared to mixing them inside resulted in two different self-assembling orientations of the cells. When they were placed on top of collagen hydrogels, a toroid ring of cells was formed. We have termed these toroids "self-organizing tissue structures". When mixed into the gels, there was contraction of the scaffold but the cells remained spread throughout the gel, other than some aggregation on the outer edge [5].

Recognizing this need we have extended our initial studies to include a battery of cell types and also bioactive matrices. To date, we have cultured no less than 10 different cell types including several types of stem cells, cardiac fibroblasts, microvascular endothelial cells and cancer cells. We have determined that no matter which cell type we use, the toroid is universally formed. The only exception to this tenet is that the numerous cancer cell lines we have cultured do not form toroids. We have extended our model to use non-animal matrices e.g. VitroGel ${ }^{\mathrm{TM}} 3 \mathrm{D}$ and PLGA gels to determine if similar pathways are activated in these cells on these matrices during toroid formation. These data coupled with high resolution imaging of key focal adhesion molecules and ECM receptors will provide the necessary pieces to provide for the integration of new simulation tools e.g. predictive models of this process to aid in guiding new bioactive materials.

As stated above the toroid is a structure that is created when cells are cultured on the surface of the collagen and take the shape of the well in which its cultured. Figure 1 shows this toroid in comparison to gels where cells are cultured within the matrix itself. What is striking is the shape of the cells forming the toroid when compared to those cells cultured inside the gel. The toroidal cells are long and thin and like the spokes of a wheel migrating towards the toroid from the outer gel. This can be seen in even greater detail using a Leica SP8 DIVE multiphoton confocal microscopy that has the capability of imaging collagen using second harmonic generation (SHG). Figure 2 shows cells in and around the toroid taken on the SP8 multiphoton microscope. This type of image begins to elucidate the movement of the cells in relationship to the collagen. Because of the second harmonic generation capabilities the collagen fibrils are easily observed. Cells appear to move through the collagen matrices by forming small channels they migrate through ending in the toroid. It appears that even though the initial cells are completely uniform over the surface of the collagen hydrogel, only the cells on the outer cortex move to the toroid in contrast to those cells in the center of the toroid which remain random in their orientation Figure 1D. 
In conclusion, our study will contribute to the modeling and computational foundation of cell behavior by providing efficient and effective integration of modeling and simulation tools with experimental research to guide the development of new biomaterial assemblies. Thus, we are uniquely positioned to make seminal advances in the field of bioactive materials and their cellular responses [6].

\section{References:}

[1] Vu LT et al, Tissue Eng Part B 21 (2014) p. 275.

[2] Fan, C. Wang, D.A., Tissue Eng Part B 23 (2017), p. 451.

[3] Sinha, R. et al, Tissue Eng Part B 23 (2017), p. 494.

[4] Gouveia, RM. et al, Tissue Eng Part A (2015), p. 321.

[5] Gourdie et al, Microsc Microanal 18 (2012), p. 99.

[6] The authors acknowledge funding from SPARC graduate research grant, Cook Biotech, FirstString Research Inc, NIH 2 P20-RR016434-06, NIH INBRE grant for South Carolina P20GM103499, R01 HL126747.

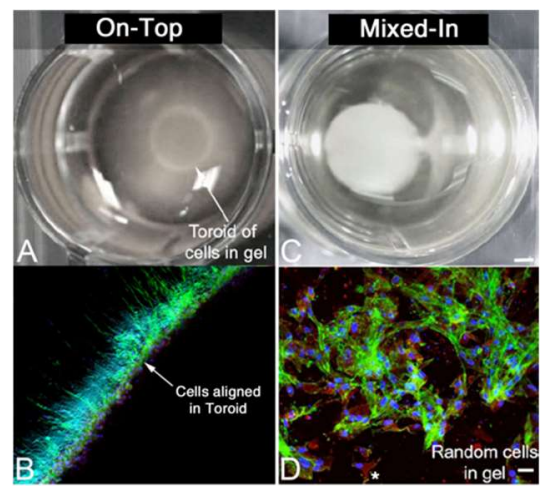

Figure 1. A) A toroid formed after LECs were seeded on top of a collagen gel. B) A segment of toroid is shown at higher magnification following immunolabeling. Note the radial alignment of cells in the toroid. C) Example of a collagen gel in which the LECs were mixed-in. D) Detail within a mixed-in collagen gel immunolabeled. Scale bars= A,C (5 mm), C,D $(10 \mu \mathrm{m})$. Blue=DAPI, Green=Phalloidin, Red=SMA

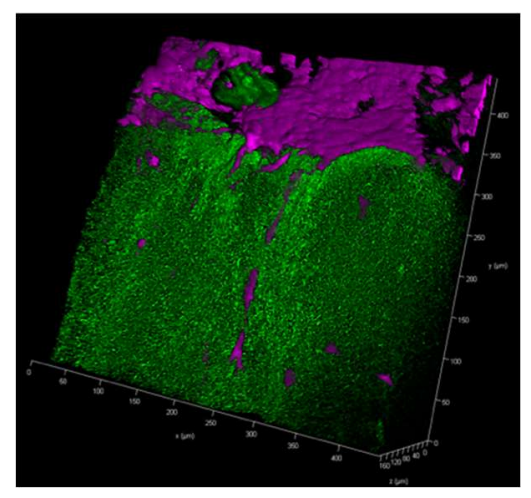

Figure 2. Multiphoton confocal image of the toroid taking with SHG. A 3-D image of the BMSCs migrating towards the toroid (cells=purple). The collagen fibirls are seen as green using SHG. The toroid (top) shows a union of cells with more migrating towards it. 